\title{
Vagal Threshold Determination during Incremental Stepwise Exercise in Normoxia and Normobaric Hypoxia
}

\author{
Filip Neuls *, Jakub Krejci ${ }^{\circ}$, Ales Jakubec, Michal Botek and Michal Valenta \\ Department of Natural Sciences in Kinanthropology, Faculty of Physical Culture, Palacky University Olomouc, \\ 77111 Olomouc, Czech Republic; jakub.krejci@upol.cz (J.K.); ales.jakubec@upol.cz (A.J.); \\ michal.botek@upol.cz (M.B.); michal.valenta@upol.cz (M.V.) \\ * Correspondence: filip.neuls@upol.cz; Tel.: +420-739-329-913
}

Received: 24 July 2020; Accepted: 14 October 2020; Published: 19 October 2020

check for updates

\begin{abstract}
This study focuses on the determination of the vagal threshold $\left(\mathrm{T}_{\mathrm{va}}\right)$ during exercise with increasing intensity in normoxia and normobaric hypoxia. The experimental protocol was performed by 28 healthy men aged 20 to 30 years. It included three stages of exercise on a bicycle ergometer with a fraction of inspired oxygen $\left(\mathrm{FiO}_{2}\right) 20.9 \%$ (normoxia), $17.3 \%$ (simulated altitude $\sim 1500 \mathrm{~m}$ ), and $15.3 \%$ $(\sim 2500 \mathrm{~m})$ at intensity associated with $20 \%$ to $70 \%$ of the maximal heart rate reserve (MHRR) set in normoxia. $\mathrm{T}_{\mathrm{va}}$ level in normoxia was determined at exercise intensity corresponding with $(\mathrm{M} \pm \mathrm{SD})$ $45.0 \pm 5.6 \%$ of MHRR. Power output at $\mathrm{T}_{\mathrm{va}}\left(\mathrm{PO}_{\mathrm{th}}\right)$, representing threshold exercise intensity, decreased with increasing degree of hypoxia (normoxia: $114 \pm 29 \mathrm{~W} ; \mathrm{FiO}_{2}=17.3 \%: 110 \pm 27 \mathrm{~W} ; \mathrm{FiO}_{2}=15.3 \%$ : $96 \pm 32 \mathrm{~W})$. Significant changes in $\mathrm{PO}_{\text {th }}$ were observed with $\mathrm{FiO}_{2}=15.3 \%$ compared to normoxia $(p=0.007)$ and $\mathrm{FiO}_{2}=17.3 \%(p=0.001)$. Consequentially, normoxic \%MHRR adjusted for hypoxia with $\mathrm{FiO}_{2}=15.3 \%$ was reduced to $39.9 \pm 5.5 \%$. Considering the convenient altitude for exercise in hypoxia, $\mathrm{PO}_{\text {th }}$ did not differ excessively between normoxic conditions and the simulated altitude of $\sim 1500 \mathrm{~m}$, while more substantial decline of $\mathrm{PO}_{\text {th }}$ occurred at the simulated altitude of $\sim 2500 \mathrm{~m}$ compared to the other two conditions.
\end{abstract}

Keywords: autonomic nervous system; vagal withdrawal; simulated altitude; exercise intensity; saturation

\section{Introduction}

Heart rate variability (HRV), i.e., the change in the time intervals between subsequent heartbeats, is an emergent property of interdependent regulatory systems that operate on different time scales to adapt to challenges and achieve optimal performance [1]. Monitoring of the HRV by the time differences of subsequent RR intervals (the time elapsed between two successive $R$ waves of the QRS signal) on the electrocardiogram (ECG) curve has been established as a useful non-invasive tool for the assessment of autonomic nervous system (ANS) activity, particularly parasympathetic (vagal) cardiac regulation [2]. Vagal activity can be mirrored by time domain parameter of the mean of the squares of the successive differences (MSSD) between adjacent RR intervals [3]. It is also associated with the respiratory modulated fluctuation of heart rate (HR) that causes a phenomenon known as respiratory sinus arrhythmia [4].

Physical exercise belongs to significant variables influencing the balance between the sympathetic and vagal activity that predominantly regulates HR with intensity and volume of exercise being the most important factors to consider [5]. Regarding increasing intensity of exercise, the accompanying rise in HR [6] has been attributed to vagal withdrawal at low to moderate exercise intensity, while the further increment in HR is induced by the rise in sympathoadrenal activity [7] as a response to the activation of hypothalamic-pituitary-adrenal axis [8]. 
HRV indexes related to cardiac vagal activity demonstrate a curvilinear decay in which the indexes decrease with increasing exercise intensity to a certain point at approximately moderate intensity [5]. After this point, the values of the vagal-related indexes can be considered almost zero and the change with a further increase in exercise intensity can be considered as negligible. The above-mentioned point occurring at a particular exercise intensity has been defined as the vagal threshold ( $T_{v a}$ ) [9]. One of the potential mechanisms of adverse clinical events may be associated with a rapid loss of vagal activity followed by sympathetic rise during exercise with a higher intensity above $\mathrm{T}_{\mathrm{va}}$ [10]. It is assumed that the determination of the vagal activity threshold (or HR corresponding with $\mathrm{T}_{\mathrm{va}}$ ) would be eventually helpful for subjects who are more prone to health complications entailed with elevated sympathoadrenal activity to minimize the risk of sudden cardiac death [11]. As reduced cardiac vagal activity accompanied with an elevated cardiac sympathetic activation has been associated with an increased risk of malignant ventricular arrhythmias or electrical instability of the heart, individuals with such cardiac complications should avoid intensities of exercise above the $\mathrm{T}_{\mathrm{va}}$ [12]. On the other hand, the physical exercise up to the $\mathrm{T}_{\mathrm{va}}$ for with preserved vagal activity is considered to be safe in the light of cardiac stress [9] with habitual physical activity being established to have a cardioprotective effect in cardiac patients [13]. Moreover, $\mathrm{T}_{\mathrm{va}}$ can be used as an indicator of the optimal exercise intensity suitable for health promotion in normal subjects [14].

Hypoxia, as characterized by a decrease in the inspired oxygen pressure [15], belongs to various environmental stressors which attenuate HRV [16]. The lower pressure of inspired oxygen in inhaled air (either terrestrial or simulated altitude) is associated with the decrease in blood oxygen saturation $\left(\mathrm{SpO}_{2}\right)$ [17]. Decreased $\mathrm{SpO}_{2}$ level has been considered to be an important factor that influences the cardiovascular compensation response in hypoxic conditions [18]. As reviewed by Oliveira et al. [19], hypoxia has been capable of generating a decrease in HRV primarily due to attenuation in vagal activity which is accompanied by relative increase in sympathoadrenal regulation. Consequentially, hypoxia leads to acceleration in the HR. This effect appears to be dependent on altitude level and barometric pressure.

Intermittent hypoxic training at simulated altitudes of $\sim 2000-2400 \mathrm{~m}$ may improve cardiovascular health and autonomic balance [20]. It should also be stressed that high altitude has become a popular leisure-time destination that is visited not only by healthy individuals but also by increasing numbers of patients with preexisting diseases. Acute hypoxia belongs to relevant factors that may represent a trigger for sudden cardiac death [21]. Pre-exposure assessment (e.g., via simulated altitude) helps to minimize risk and detect contraindications to high-altitude exposure or help with preconditioning [22]. A response to exposition to hypoxic conditions is highly individual; hence, it is also desirable to individualize recommendations related to possibilities of exposition to hypoxia including its duration and $\mathrm{FiO}_{2}$ and or degree of personal cardiac health, for example by means of monitoring the changes in the HRV [23].

Taken together, the synergistic effect of the two stress stimuli, exercise and hypoxia, employs assumed influence on the reduction in cardiac vagal activity and a relative rise in sympathoadrenal activity, depending on the exercise intensity and degree of hypoxia Therefore, this study was aimed to determine $\mathrm{T}_{\mathrm{va}}$ level during increasing physical load in normoxia and two normobaric simulated altitudes ( $1500 \mathrm{~m}$ and $\sim 2500 \mathrm{~m})$ in a sample of healthy men with a possibility of subsequent suggestions how to define an adequate altitude or exercise intensity for individuals with an elevated risk of cardiac complications. Thus, we were interested in the assessment of responses of selected physiological variables to the combined effect of exercise intensity and hypoxia at the $\mathrm{T}_{\mathrm{va}}$ level.

\section{Materials and Methods}

\subsection{Subjects}

The sample consisted of 28 healthy male volunteers aged 20 to 30 years. They were recruited among students of sports sciences, and the participation was limited only to non-smokers. The original 
number of participants $(n=30)$ was reduced by two persons who did not complete all requirements of the study. The somatic and physiological characteristics of the experimental group are presented in Table 1.

Table 1. Anthropological and physiological characteristics of the participants $(n=28)$.

\begin{tabular}{cccc}
\hline Variable & $\mathbf{M} \pm$ SD & Variable & M \pm SD \\
\hline Age (years) & $23.5 \pm 2.0$ & $\mathrm{FEV}_{1}(\mathrm{~L})$ & $4.7 \pm 0.7$ \\
Body mass $(\mathrm{kg})$ & $74.2 \pm 6.9$ & $\mathrm{FEV}_{1}$ predicted $(\%)$ & $108 \pm 12$ \\
Body height $(\mathrm{cm})$ & $176.7 \pm 7.2$ & $\mathrm{VO}_{2} \mathrm{max}\left(\mathrm{ml} \cdot \mathrm{kg}^{-1} \cdot \mathrm{min}^{-1}\right)$ & $48.2 \pm 6.4$ \\
BMI $\left(\mathrm{kg} \cdot \mathrm{m}^{-2}\right)$ & $23.8 \pm 1.9$ & HRmax $\left(\right.$ beats $\left.\cdot \mathrm{min}^{-1}\right)$ & $191.6 \pm 7.2$ \\
Body fat $(\%)$ & $10.9 \pm 3.5$ & HRrest (beats $\left.\cdot \mathrm{min}^{-1}\right)$ & $56.6 \pm 5.2$ \\
FFM $(\mathrm{kg})$ & $66.0 \pm 6.2$ & MHRR (beats $\left.\cdot \mathrm{min}^{-1}\right)$ & $134.9 \pm 7.6$ \\
VC $(\mathrm{L})$ & $5.3 \pm 0.8$ & Pmax $\left(\mathrm{W} \cdot \mathrm{kg}^{-1}\right)$ & $4.6 \pm 0.5$ \\
VC predicted $(\%)$ & $98 \pm 11$ & & \\
\hline
\end{tabular}

All values were obtained in normoxic conditions. M: mean, SD: standard deviation, BMI: body mass index, FFM: fat-free mass, VC: vital capacity of lungs, $\mathrm{FEV}_{1}$ : forced expiratory volume in $1 \mathrm{~s}, \mathrm{VO}_{2}$ max: maximal oxygen uptake, HRmax: maximal heart rate, HRrest: resting heart rate, MHRR: maximal heart rate reserve, Pmax: maximal power output.

This study project was approved by the Ethics Committee of the Faculty of Physical Culture, Palacky University in Olomouc (Czech Republic) under the identification number 80/2018. All subjects gave written informed consent in accordance with the Declaration of Helsinki and filled in a form of "Lausanne Recommendations: sudden cardiovascular death in sport" [24]. The participants were informed in detail about the aims and scope of the study with the possibility to withdraw from the study at any time.

\subsection{Experimental Protocol}

All processes associated with the experiment were performed in the laboratory of exercise physiology at the Faculty of Physical Culture, Palacky University in Olomouc. All measurements were conducted between 8:00 a.m. and 1:00 p.m. Ambient temperature in the laboratory ranged between 22 and $24{ }^{\circ} \mathrm{C}$. The altitude of the laboratory was $260 \mathrm{~m}$ above sea level $\left(\mathrm{FiO}_{2}=20.9 \%\right)$. The participants were instructed that factors possibly influencing their ANS should be avoided from two hours (e.g., caffeine or tea intake, eating, specific medications or substances) up to one day (e.g., strenuous physical activities) prior to particular measurements.

The study consisted of five main stages, as described graphically in Figure 1 (see below a detailed description of instruments and techniques used). A delay of at least two days between each measurement stage was respected.

The first stage comprised of anthropometry and body composition measures, measuring of resting HR (HRrest) and HRV, spirometry (vital capacity and forced expiratory volume in $1 \mathrm{~s}$ ), and the participants underwent maximal incremental test on a bicycle ergometer to assess maximal oxygen uptake $\left(\mathrm{VO}_{2} \max \right)$, maximal heart rate (HRmax), maximal power output (Pmax), and ventilation parameters (minute ventilation $-\mathrm{VE}$, tidal volume $-\mathrm{Vt}$, and breath frequency $-\mathrm{Bf}$ ). All measurements were performed in normoxia.

After the determination of HRrest and HRmax, maximal heart rate reserve (MHRR) was computed for each subject according to a formula MHRR $=$ HRmax - HRrest [25]. Consequentially, we calculated individual heart rates corresponding with $20 \%, 30 \%, 40 \%, 50 \%, 60 \%$, and $70 \%$ of MHRR using a formula HRtarget $=$ HRrest + MHRR $\times \mathrm{K} / 100$, where $\mathrm{K}$ is a percentage coefficient.

Stage 2 was performed to set and record individual power outputs and cadences (pedaling frequencies) needed to reach particular target HRs matching previously computed values of $20 \%$ to $70 \%$ of MHRR. For this purpose, power output and cadence were continuously adjusted during pedaling to reach required HR firstly at $20 \%$ of MHRR and to keep it steady (i.e., \pm 2 beats. $\mathrm{min}^{-1}$ ) for at least one minute before continuing to the next HR accommodating 30\% of MHRR. This process was performed 
repeatedly up to HR matching 70\% of MHRR. The setting procedure was performed in normoxia. Recorded values of power output (watts) and pedaling frequencies associated with particular target $\mathrm{HR}$ at 20 to $70 \%$ of MHRR were used to create an individual template protocol for testing in the subsequent three experimental stages performed in normoxia and two simulated altitudes of $\sim 1500 \mathrm{~m}$ and $\sim 2500 \mathrm{~m}$.

Stages 3 to 5 followed the same procedure consisting of experimental test on the bicycle ergometer, starting with the preset power output and cadence corresponding with $20 \%$ of MHRR determined during the setting process in stage 2 . Each step of the experimental test (successively at 20\%, 30\%, 40\%, $50 \%, 60 \%$, and $70 \%$ of MHRR) lasted $3 \mathrm{~min}$ (expected sufficient time to reach a steady state within each step [26]), so the total duration of the test was $18 \mathrm{~min}$. Order of the stages 3, 4, and 5 was randomized for each participant using a computer-generated permutation table. This process was not disclosed to the participants as they were not informed about the degree of normoxia/hypoxia in which they performed the testing.

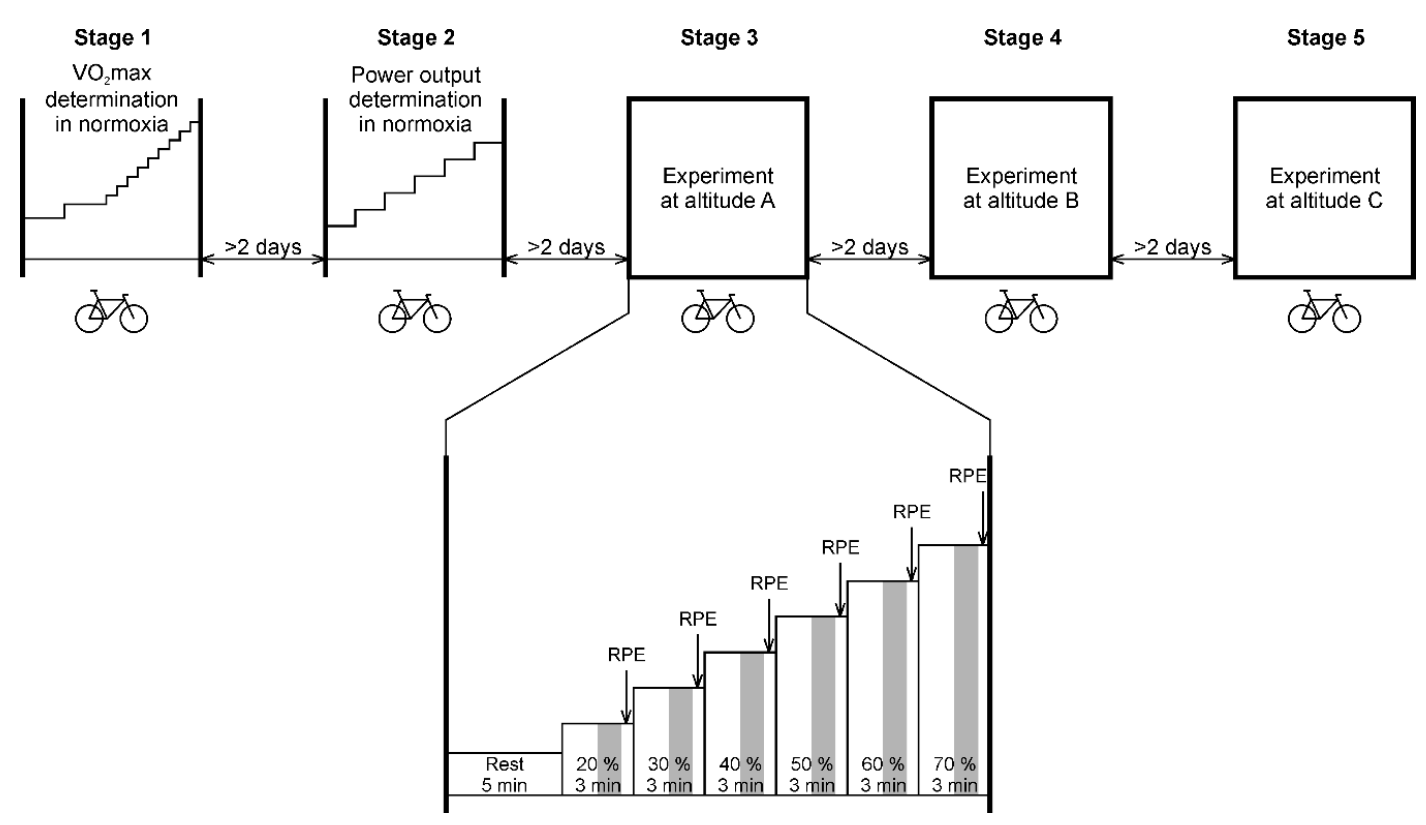

Figure 1. Course of experimental protocol. Altitudes marked A, B, C were randomly assigned to normoxia and two levels of normobaric hypoxia. Gray rectangles denote 1-min segments for calculating average values of heart rate variability, oxygen saturation, and ventilatory measures. RPE: rating of perceived exertion, $\mathrm{SpO}_{2}$ : arterial oxygen saturation.

\subsection{Anthropometrical Measurement}

Subjects underwent the basic anthropometrical measurement. Body height was measured by a stadiometer. Body mass, percentage of body fat and fat-free mass were determined using bio-impedance analysis (Tanita BC-418 MA, Tanita, Tokyo, Japan).

\subsection{Resting Spirometry}

The spirometry test with calibrated instruments (Spirostik with Blue Cherry software; Geratherm Respiratory, Bad Kissingen, Germany) was performed to assess individual vital capacity (VC) and forced expiratory volume in one second $\left(\mathrm{FEV}_{1}\right)$. These values were also recounted to the values predicted according to the body surface area (\%).

\subsection{Maximal Incremental Testing}

The maximal incremental test was performed on the bicycle ergometer Ergoline 800 (Ergoline, Bitz, Germany). The exercise protocol consisted of an 8-min warm-up period (4 min at $120 \mathrm{~W}$ and $4 \mathrm{~min}$ at $160 \mathrm{~W}$, both with $70 \mathrm{rpm}$ cadence) followed by 1-min incremental steps, starting at $220 \mathrm{~W}$ 
and increasing by $20 \mathrm{~W}$ every minute until exhaustion. Cadence increased arbitrarily following the individualized needs of participants to reach the maximum.

Breath-by-breath ventilation and gas exchange were measured (Ergostik with Blue Cherry software; Geratherm Respiratory, Bad Kissingen, Germany) during the exercise with the data averaged to $30 \mathrm{~s}$ for analysis. Gas and flow analyzers were recalibrated before each testing using gases of known concentration and a 3-L calibration syringe.

The following criteria were used to document that $\mathrm{VO}_{2}$ max was achieved: (1) a lack of increase in $\mathrm{VO}_{2}$ upon an increase in work rate, and (2) respiratory exchange ratio $>1.10 . \mathrm{VO}_{2}$ max was recorded as the highest $\mathrm{VO}_{2}$ value in the final $30 \mathrm{~s}$ of the test. Heart rate response was measured continuously using a chest strap (Polar Electro Oy, Kempele, Finland). HRmax was defined as the highest HR recorded during the test. The relative value of maximal power output (Pmax) was determined as the highest wattage reached and maintained during the last $30 \mathrm{~s}$ of the test, divided by individual body mass.

\subsection{Hypoxic Chamber}

Normobaric hypoxia conditions were created using a hypoxic chamber and HR-1470 generator of hypoxic air (Hypoxie group, Prague, Czech Republic). In this study, two levels of normobaric hypoxia with $\mathrm{FiO}_{2}=17.3 \%$ and $15.3 \%$ were used, which correspond to simulated altitudes of $\sim 1500 \mathrm{~m}$ and $\sim 2500 \mathrm{~m}$, respectively. Volume of the chamber was $45.5 \mathrm{~m}^{3}$ (length: $7.0 \mathrm{~m}$; width: $2.5 \mathrm{~m}$; height: $2.6 \mathrm{~m}$ ). The generator separates compressed air into nitrogen and oxygen fractions using a system of a hollow fiber membrane. As an output of this separation process, oxygen-reduced air flows into the chamber. Required $\mathrm{FiO}_{2}$ inside the chamber was continuously maintained by a controlling system of inlet/outlet valves and calibrated sensors. Carbon dioxide $\left(\mathrm{CO}_{2}\right)$ concentration was kept under $1500 \mathrm{ppm}(0.15 \%)$ by its regular airing out after each experimental test. Relative humidity of approximately 30 to $40 \%$ was maintained in the chamber by a common commercial humidifier.

\subsection{Heart Rate and Heart Rate Variability Measurement}

RR intervals were measured continuously during the experimental test on bicycle ergometer using a Polar V800 heart rate monitor (Polar, Kempele, Finland) which was proven to be a valid tool for measuring the RR interval [27]. RR records were transferred to a computer using the Polar Flow cloud service. Artefacts (ectopic beats, missing beats, etc.) were identified by visual inspection of RR intervals and simply deleted because the deletion method provided the best overall performance [28]. The MSSD value and average HR value were calculated from a 1-min segment that started $1.5 \mathrm{~min}$ from the beginning of the 3-min step (Figure 1).

\subsection{Oxygen Saturation Measurement}

Arterial oxygen saturation $\left(\mathrm{SpO}_{2}\right)$ was measured continuously during the experimental test using a Nonin Avant 4000 pulse oximeter (Nonin Medical, Minneapolis, MN, USA) with a sensor placed on the left middle finger. $\mathrm{SpO}_{2}$ was measured at a sampling frequency of $1.0 \mathrm{~Hz}$, and the average value was calculated using a 1-min segment that started $1.5 \mathrm{~min}$ from the beginning of the 3-min step (Figure 1).

\subsection{Ventilation Measurement}

Breath-by-breath ventilation was continuously measured (Ergostik with Blue Cherry software; Geratherm Respiratory, Bad Kissingen, Germany) also during all three experimental stages 3 to 5 . For further statistical processing, the average values of $\mathrm{Vt}, \mathrm{Bf}$, and $\mathrm{VE}$ were calculated using a 1-min segment that started $1.5 \mathrm{~min}$ from the beginning of the 3-min step (Figure 1).

\subsection{Rating of Perceived Exertion}

Rating of perceived exertion (RPE) was applied as a psychophysiological measure during the three experimental testing stages (10 s before the end of each 3-min step). For this purpose, the revised Borg's 
category-ratio scale ( 0 to 10 scale) was used [29]. The subjects were instructed to express a numerical value for their RPE with the help of text descriptors.

\subsection{Vagal Threshold Determination}

Vagal threshold values were calculated using a custom application written in MATLAB 8.4 (MathWorks, Natick, MA, USA). The algorithm used was described in detail by Botek et al. [30]. The input to the algorithm was a set of 6 MSSD values measured at a six different power output values. In order to be able to process subjects with different levels of physical fitness, power outputs in watts were set individually to correspond to $20 \%, 30 \%, 40 \%, 50 \%, 60 \%$, and $70 \%$ MHRR in normoxia (stage 2). The individual power outputs were kept the same during normoxia/hypoxia stages 3 to 5 and were labelled as the percentage of MHRR in normoxia. The algorithm split the set into two subsets and calculated $\mathrm{T}_{\mathrm{va}}$ as the intersection point of two regression lines. In the beginning, the first subset contained 2 MSSD values obtained on power outputs corresponding to $20 \%$ and $30 \% \mathrm{MHRR}$, and the second subset contained 5 MSSD values obtained on power outputs corresponding to $30 \%$ to $70 \%$ MHRR. Regression lines were calculated using least-square method and a condition was tested whether the second regression line has a decreasing slope and lies above the zero level. If the condition was not met, an MSSD value corresponding to the next output power was added to the first subset, and this MSSD value was removed from the second subset. Regression lines were recalculated and a new intersection point was calculated. This iterative procedure was repeated until the condition was met. An illustrative example of calculating the vagal threshold is shown in Figure 2. The algorithm provided power output value $\left(\mathrm{PO}_{\text {th }}\right)$ at which the vagal threshold was found and a corresponding MSSD threshold value $\left(M_{S S D}{ }_{\text {th }}\right) . \mathrm{PO}_{\text {th }}$ was expressed in watts and also approximated as a percentage of MHRR related to normoxia. The threshold values of other variables $\left(\mathrm{HR}_{\mathrm{th}}, \mathrm{SpO}_{2 \mathrm{th}}, \mathrm{VE}_{\mathrm{th}}, \mathrm{BF}_{\text {th }}, \mathrm{Vt}_{\text {th }}\right.$, and $\left.\mathrm{RPE}_{\text {th }}\right)$ were calculated by linear interpolation using two consecutive steps in the vicinity of the $\mathrm{PO}_{\text {th }}$.
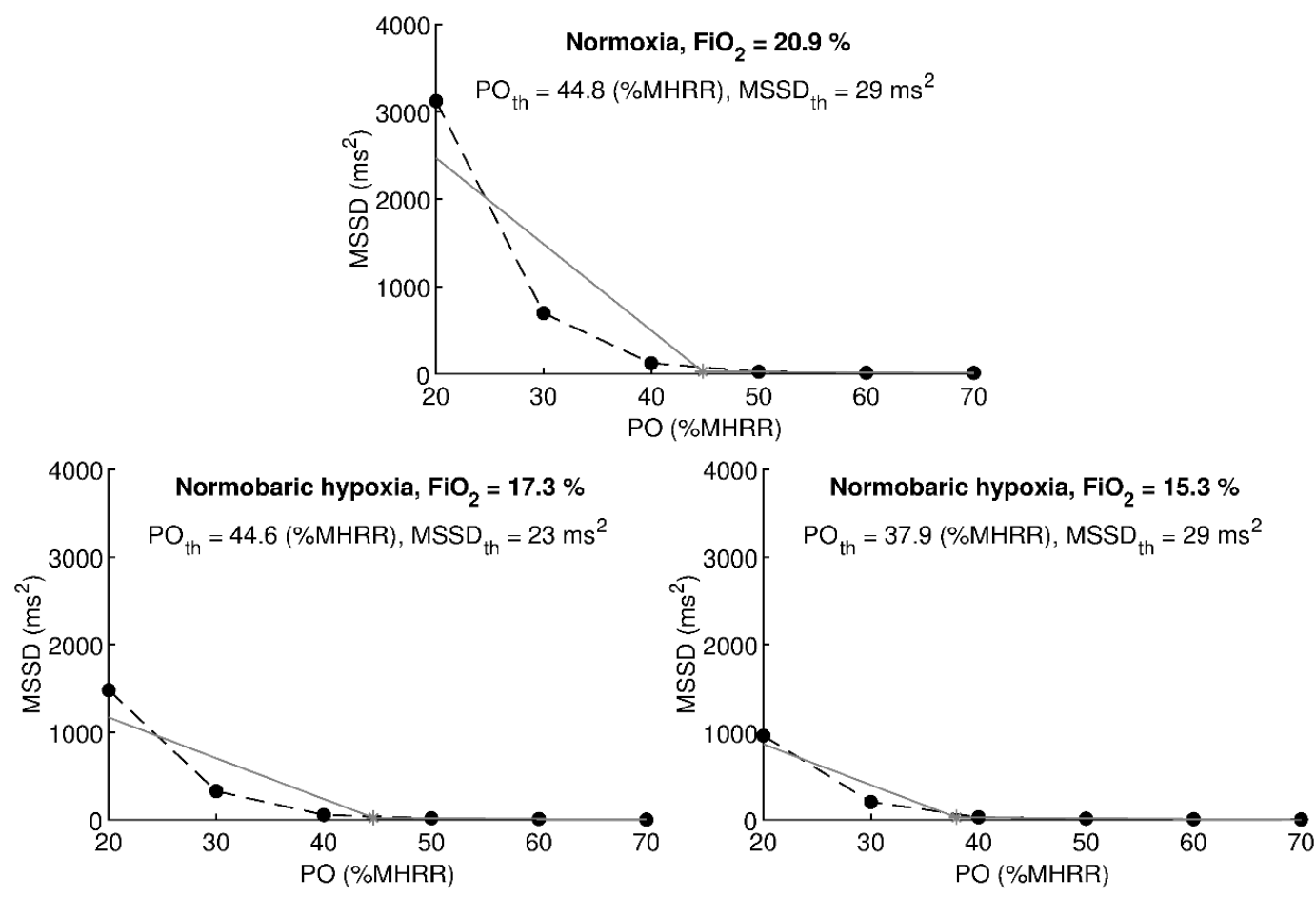

Figure 2. Illustrative example of calculating vagal threshold of one chosen participant at normoxia and two levels of normobaric hypoxia. Black circles and dashed line denote measured data. Grey lines denote regression lines. Grey asterisk indicates vagal threshold point. PO: power output corresponding to a percentage of maximal heart rate reserve in normoxia, MSSD: the mean of the squares of the successive differences between adjacent RR intervals, th: threshold. 


\subsection{Statistical Analysis}

To verify that RR intervals in the 1-min segment were stationary, the segment was divided into $30 \mathrm{~s}$ sub-segments and values between adjacent sub-segments were compared using an analysis of variance (ANOVA) for repeated measures with three fixed factors (altitude, load, and sub-segment order); however, only the significance of the order factor was considered.

Particular vagal thresholds were determined using the algorithm described above and threshold values of studied variables were calculated. Data normality was verified using the Kolmogorov-Smirnov test and $p<0.05$ was considered statistically significant. The Kolmogorov-Smirnov test rejected the normality of $\mathrm{PO}_{\text {th }}, \mathrm{MSSD}_{\mathrm{th}}, \mathrm{VE}_{\mathrm{th}}$, and $\mathrm{RPE}_{\mathrm{th}}$, so the threshold values between normoxia/hypoxia were compared using a set of 3 separate Wilcoxon tests. Bonferroni adjustment was used to control the Type 1 statistical error, therefore $p<0.05 / 3$ was considered statistically significant. Potential predictors of $\mathrm{PO}_{\text {th }}$ were searched using Pearson's correlation coefficient.

Data are presented as arithmetic mean \pm standard deviation. Statistical analyses were performed using MATLAB 8.4 (MathWorks, Natick, MA, USA) and the STATISTICA 13.4 (StatSoft, Tulsa, OK, USA). A priori power analysis considering a Wilcoxon test was performed using $G^{*}$ Power 3.1.9.7 (Heinrich-Heine-Universität, Düsseldorf, Germany) with parameters $d_{z}=0.8, \alpha=0.017$, and $\beta=0.20$. The minimal sample size resulted in 21 subjects.

\section{Results}

The 1-min segments were divided into $30 \mathrm{~s}$ segments and ANOVA did not reveal any statistically significant difference between adjacent $30 \mathrm{~s}$ segments for HR $(p=0.999)$ and MSSD after the logarithmic transformation $(p>0.999)$. This indicates that a pause of $1.5 \mathrm{~min}$ after the start of each step was sufficient to achieve RR intervals to be stationary.

The threshold values of physiological and psychological variables in normoxia and normobaric hypoxia of $\mathrm{FiO}_{2}=17.3 \%$ and $15.3 \%$ are given in Table 2 . The hypoxia of $17.3 \%$ compared to normoxia did not induce statistically significant changes in all studied variables (all $p \leq 0.109$ ) except $\mathrm{SpO}_{2}$ $(p<0.001)$. The hypoxia of $15.3 \%$ compared to normoxia significantly decreased $\mathrm{PO}_{\text {th }}$ expressed in watts $(p=0.007), \mathrm{PO}_{\text {th }}$ expressed as \%MHRR in normoxia $(p=0.001)$, and $\mathrm{SpO}_{2 \text { th }}(p<0.001)$. These decreases were also statistically significant when comparing hypoxia of $15.3 \%$ with hypoxia of $17.3 \%$ (all $p \leq 0.001$ ). The threshold values $\mathrm{MSSD}_{\text {th }}, \mathrm{HR}_{\mathrm{th}}, \mathrm{VE}_{\mathrm{th}}, \mathrm{BF}_{\mathrm{th}}, \mathrm{Vt}_{\mathrm{th}}, \mathrm{RPE}_{\text {th }}$ did not change significantly when hypoxia of $15.3 \%$ was compared to normoxia (all $p \leq 0.126$ ) or hypoxia of $17.3 \%$ (all $p \leq 0.066$ ).

Table 2. Threshold values of physiological and psychometric variables at normoxia $\left(\mathrm{FiO}_{2}=20.9 \%\right)$ and normobaric hypoxia $\left(\mathrm{FiO}_{2}=17.3 \%\right.$ and $\left.15.3 \%\right)$.

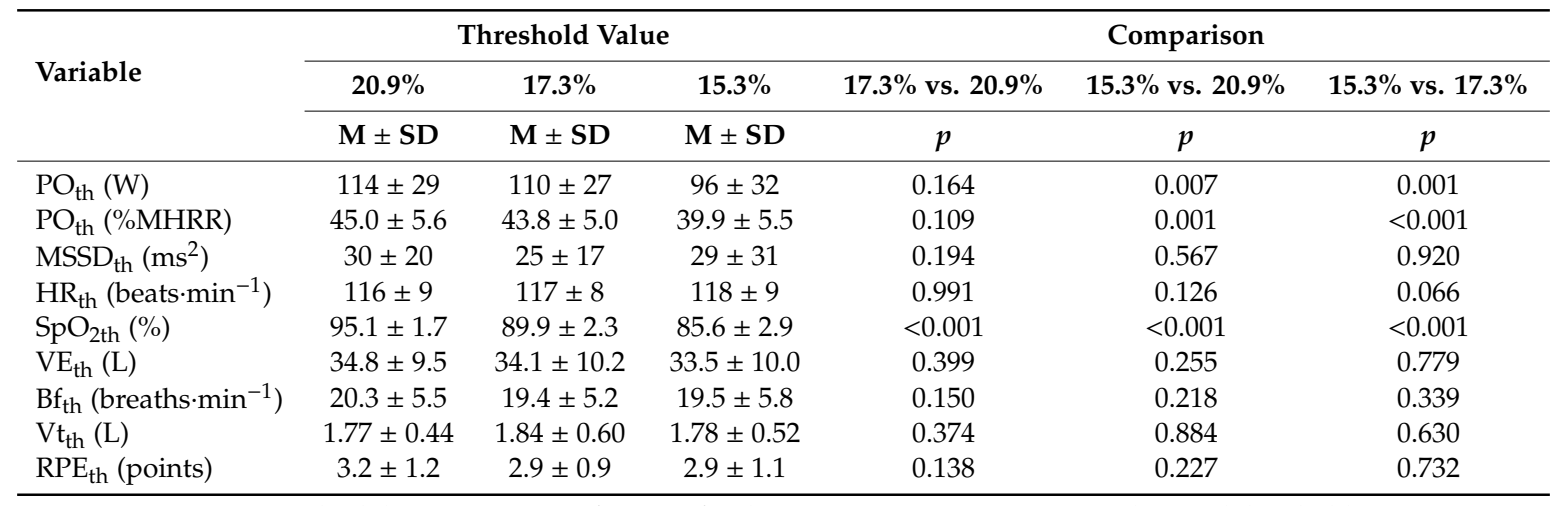

M: mean, SD: standard deviation, $p$ : significance of Wilcoxon test, PO: power output, th: vagal threshold, \%MHRR: percentage of maximal heart rate reserve in normoxia, MSSD: the mean of the squares of the successive differences between adjacent $\mathrm{RR}$ intervals, $\mathrm{HR}$ : heart rate $\mathrm{SpO}_{2}$ : arterial oxygen saturation, VE: minute ventilation, Bf: breathing frequency, Vt: tidal volume, RPE: rate of perceived exertion. 
Individual differences in $\mathrm{PO}_{\text {th }}$ between hypoxia of $15.3 \%$ and normoxia were significantly $(p=0.010)$ associated with individual differences in VE between hypoxia of $15.3 \%$ and normoxia observed at exercise step corresponding to $40 \%$ MHRR in normoxia (Figure 3). The differences in $\mathrm{PO}_{\text {th }}$ were not significantly associated with body mass, body fat, VC, FEV1, Pmax, and $\mathrm{VO}_{2} \max$ (all $p \geq 0.059$ ) or differences in $\mathrm{SpO}_{2}, \mathrm{BF}, \mathrm{Vt}$, and $\mathrm{RPE}$ (all $p \leq 0.234$ ).

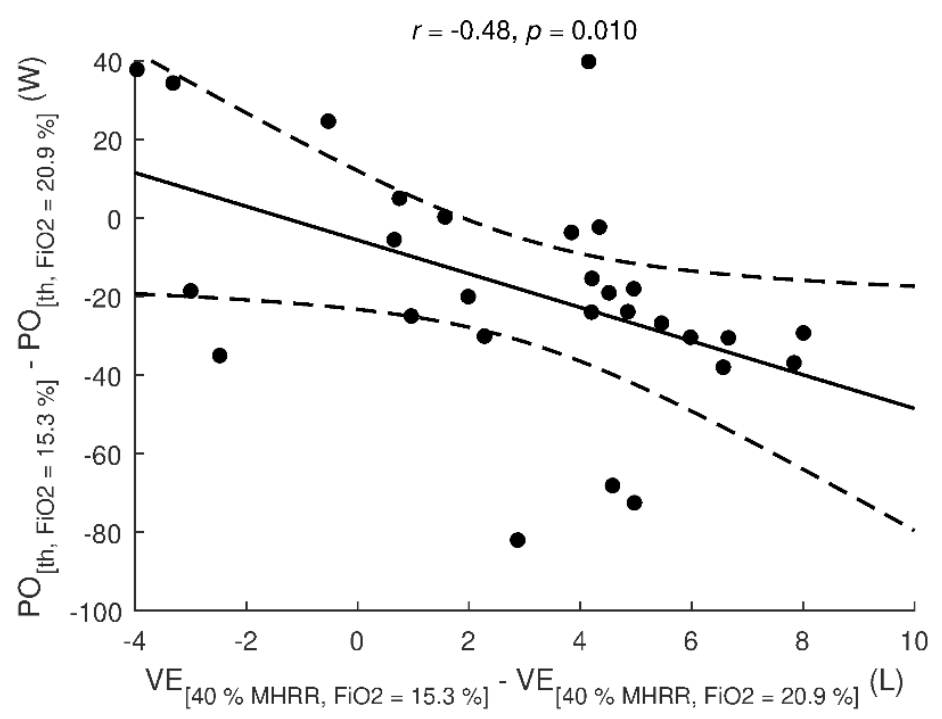

Figure 3. Association between changes in ventilation at step corresponding to $40 \% \mathrm{MHRR}$ and changes in power output at vagal threshold. Changes were calculated as the value at hypoxia $\left(\mathrm{FiO}_{2}=15.3 \%\right)$ minus the value at normoxia $\left(\mathrm{FiO}_{2}=20.9 \%\right)$. Dashed lines denote 0.95-confidence interval. $r$ : Pearson correlation coefficient, $p$ : significance of correlation, VE: minute ventilation, PO: power output, MHRR: maximal heart rate reserve, th: vagal threshold.

\section{Discussion}

The primary purpose of this study was to compare changes in vagal activity during exercise with increasing intensity in normoxia and two simulated conditions of normobaric hypoxia. Previous studies were oriented mostly on assessing the $\mathrm{T}_{\mathrm{va}}$ level in normoxia $[9,14,30]$ or vagal activity in various hypoxic conditions at rest (see review of Oliveira et al. [19]) so the main research question was focused on possible shifts of selected physiological variables determined at $\mathrm{T}_{\mathrm{va}}$ level during incremental stepwise exercise.

The results of this study show that $\mathrm{T}_{\mathrm{va}}$ level did not differ excessively between normoxic conditions ( $\mathrm{PO}_{\text {th }}$ of $114 \mathrm{~W}$ corresponding with $45.0 \%$ of MHRR in normoxia) and normobaric hypoxia with $\mathrm{FiO}_{2}=17.3 \%\left(\mathrm{PO}_{\text {th }}\right.$ of $110 \mathrm{~W}$ corresponding with $43.8 \%$ of MHRR in normoxia). Significant changes in $\mathrm{T}_{\mathrm{va}}$ level occurred at normobaric hypoxia with $\mathrm{FiO}_{2}=15.3 \%\left(\mathrm{~T}_{\mathrm{va}}\right.$ at $96 \mathrm{~W}$ corresponding with $39.9 \%$ of MHRR in normoxia) compared to normoxia and normobaric hypoxia with $\mathrm{FiO}_{2}=17.3 \%$. To be more specific, $\mathrm{HR}$ at the $\mathrm{T}_{\mathrm{va}}$ remained comparable (116-118 beats. $\left.\mathrm{min}^{-1}\right)$ in all the three conditions while $\mathrm{PO}_{\text {th }}$ matching the "algorithm-determined" $\mathrm{HR}_{\text {th }}$ decreased with decreasing $\mathrm{FiO}_{2}$. Initial exercise intensity corresponding with the vagal withdrawal in normoxia is in full accordance with previous findings of Botek et al. [30] except for $\mathrm{HR}$ at $\mathrm{T}_{\mathrm{va}}$, which was lower in their study of $n=10$ age-matching subgroup of men (112 beats. $\left.\mathrm{min}^{-1}\right)$. However, they used a treadmill instead of a bicycle ergometer in their experimental protocol. Similarly, Hautala et al. [31] considered approximately $40 \%$ of $\mathrm{VO}_{2} \mathrm{max}$ as the exercise intensity, where increased sympathetic activation starts to dominate after vagal withdrawal. Shibata et al. [9] assessed $\mathrm{T}_{\mathrm{va}}$ in a sample of 43 middle-aged obese women. In their study, the sympathovagal conversion occurred at $114 \pm 9$ beats. $\mathrm{min}^{-1}$. Oshima et al. [14] found in their study of $n=63$ normal subjects that the HR at the $\mathrm{T}_{\mathrm{va}}$ was $112 \pm 13$ beats. $\mathrm{min}^{-1}$ when using a ramp exercise test with a bicycle ergometer but their sample was markedly heterogeneous (men and women 
of various ages). The authors defined the criterion of the $T_{\mathrm{va}}$ as MSSD $<25 \mathrm{~ms}^{2}$. The values of MSSD in our study varied between $25-30 \mathrm{~ms}^{2}$ while no significant differences were found between normoxic and hypoxic conditions.

Vagal activity with exercise intensities above the $T_{\text {va }}$ can be suggested as negligible with minimal regulatory impact on the cardiovascular system. Iwasaki et al. [32] performed a study focused on a similar degree of normobaric hypoxia (stepwise decreases from $21 \%$ to $15 \%$ of $\mathrm{FiO}_{2}$ ) at rest. They found that acute exposure to normobaric mild hypoxia $\left(\mathrm{FiO}_{2} \geq 15 \%\right)$ induced a significant shift of the autonomic balance towards cardiac sympathetic dominance resulting in an increased HR. In general, vagal withdrawal and a relative dominance of sympathetic activity in hypoxia seems to be governed by the arterial chemoreflex [33]. However, there are probably multiple pathways controlling cardiovascular response to hypoxia involved. They include peripheral chemoreceptors, arterial baroreceptors, central nervous system hypoxic response, and lung stretch receptors [34].

Regarding $\mathrm{SpO}_{2}$ as a relevant factor influencing the HRV [16], its values at $\mathrm{T}_{\mathrm{va}}$ significantly differed in our study between all the three experimental stages (normoxia and both hypoxic conditions). According to Rojas-Camayo et al. [35], $\mathrm{SpO}_{2}$ in healthy individuals is reduced more evidently at altitudes over $2500 \mathrm{~m}$ but the effect of exercise was not taken into account. Woorons et al. [36] suggest that moderate exercise in hypoxia induces a greater arterial desaturation in trained than untrained men with the difference starting at $\mathrm{FiO}_{2}=0.154$ and below.

One of the acute responses of the body to decreasing $\mathrm{SpO}_{2}$ [37] as well as to exercise [6] is an increase in VE which appears to ensure adequate delivery of oxygen to tissues. However, we did not find any considerable differences in ventilation (neither in its components, i.e., tidal volume and respiratory rate) at the level of $\mathrm{T}_{\mathrm{va}}$. It allows us to claim that exercise intensity about $40-45 \%$ of MHRR adjusted for normoxia did not elicit any substantial ventilation changes in hypoxia up to $\sim 2500 \mathrm{~m}$. As shown in Figure 3, lower VE at exercise intensity corresponding to $40 \%$ of normoxic MHRR is moderately associated with higher power output. Thus, higher VE would probably mean a decrease in vagal activity and termination of respiratory sinus arrhythmia [38].

As mentioned before, $\mathrm{T}_{\mathrm{va}}$ can be used as an indicator of the "safe" exercise intensity suitable for health promotion in normal subjects [14]. Shibata et al. [9] claim that $\mathrm{T}_{\mathrm{va}}$ may also be recommended for obese people who might possess a lower cardiac sympathovagal balance. Shiraishi et al. [39] found that $\mathrm{T}_{\mathrm{va}}$ was strongly correlated with the ventilatory threshold in a subset of patients with myocardial infarction as well as healthy subjects when using real-time HRV analysis during ramp exercise protocol. Thus, we suggest that our study will be helpful to create a standardized procedure, which would enable setting individualized recommendations for the exercise intensity (or physical activity intensity, in general) for various groups of people in need of HR control during physical load in hypoxic conditions in order to maintain cardiac vagal protection.

As a main limitation of the study, we consider the fact that only hypoxic chamber without the possibility of creating hypobaric conditions was used. Moreover, our participants were exposed only to acute short-term hypoxic conditions so the response might be different if longer pre-acclimatization period was involved. We were also limited in possibilities to assess oxygen consumption during the experimental stages because used system of a gas analyzer and associated software was limited to work accurately in the hypoxic air. A further limitation is that we were oriented predominantly on vagal activity with the omission of sympathoadrenal system activity.

\section{Conclusions}

Exercise intensity corresponding with the withdrawal in vagal activity was $\sim 45 \%$ of MHRR in normoxia. Considering the convenient altitude for exercise in hypoxia, $\mathrm{PO}_{\text {th }}$ did not differ significantly between normoxic conditions and $\mathrm{FiO}_{2}=17.3 \%$ (simulation of $1500 \mathrm{~m}$ ). More substantial decline of the $\mathrm{PO}_{\text {th }}$ occurred in $\mathrm{FiO}_{2}=15.3 \%$ (simulation of $\sim 2500 \mathrm{~m}$ ) when compared to the other two conditions. Consequentially, $\mathrm{T}_{\mathrm{va}}$ adjusted for normobaric hypoxia with $\mathrm{FiO}_{2}=15.3 \%$ declined to $\sim 40 \%$ of $\mathrm{MHRR}$. 
Further studies performed in samples with different characteristics according to age, sex, or type of health issues are recommended.

Author Contributions: Conceptualization, M.B., J.K., and F.N.; methodology, M.B., F.N., and A.J.; software, J.K.; validation, F.N., A.J., and M.V.; formal analysis, F.N. and A.J.; investigation, F.N. and M.V.; resources, F.N.; data curation, J.K., A.J., and F.N.; writing-original draft preparation, F.N.; writing-review and editing, F.N., J.K., and M.B.; visualization, J.K.; supervision, M.B.; project administration, F.N., A.J., and M.V.; funding acquisition, M.V. All authors have read and agreed to the published version of the manuscript.

Funding: This study is a part of an internal grant funded by the Palacky University in Olomouc entitled "Determination of vagal threshold in normobaric hypoxia" (IGA_FTK_2019_003).

Acknowledgments: The authors would like to thank all of the participants for their contribution and mainly our assistant Wayne Zaparka for organizing them.

Conflicts of Interest: The authors have no conflict of interest. The funders had no role in the design of the study; in the collection, analyses, or interpretation of data; in the writing of the manuscript, or in the decision to publish the results.

\section{References}

1. Shaffer, F.; McCraty, R.; Zerr, C.L. A healthy heart is not a metronome: An integrative review of the heart's anatomy and heart rate variability. Front. Physiol. 2014, 5, 1040. [CrossRef] [PubMed]

2. Acharya, U.R.; Joseph, K.P.; Kannathal, N.; Lim, C.M.; Suri, J.S. Heart rate variability: A review. Med. Biol. Eng. Comput. 2006, 44, 1031-1051. [CrossRef] [PubMed]

3. Aubert, A.E.; Seps, B.; Beckers, F. Heart rate variability in athletes. Sports Med. 2003, 33, 889-919. [CrossRef]

4. Yasuma, F.; Hayano, J. Respiratory sinus arrhythmia: Why does the heartbeat synchronize with respiratory rhythm? Chest 2004, 125, 683-690. [CrossRef] [PubMed]

5. Michael, S.; Graham, K.S.; Davis, G.M. Cardiac autonomic responses during exercise and post-exercise recovery using heart rate variability and systolic time intervals-A review. Front. Physiol. 2017, 8, 301. [CrossRef]

6. Åstrand, P.O.; Rodahl, K.; Dahl, H.A.; Strømme, S.B. Textbook of Work Physiology: Physiological Bases of Exercise; Human Kinetics: Champaign, IL, USA, 2003.

7. Perini, R.; Veicsteinas, A. Heart rate variability and autonomic activity at rest and during exercise in various physiological conditions. Eur. J. Appl. Physiol. 2003, 90, 317-325. [CrossRef]

8. Chrousos, G.P.; Gold, P.W. The concepts of stress and stress system disorders. JAMA 1992, 267, $1244-1252$. [CrossRef]

9. Shibata, M.; Moritani, T.; Miyawaki, T.; Hayashi, T.; Nakao, K. Exercise prescription based upon cardiac vagal activity for middle-aged obese women. Int. J. Obes. 2002, 26, 1356-1362. [CrossRef]

10. Tulppo, M.P.; Mäkikallio, T.H.; Seppänen, T.; Laukkanen, R.T.; Huikuri, H.V. Vagal modulation of heart rate during exercise: Effects of age and physical fitness. Am. J. Physiol. 1998, 274, H424-H429. [CrossRef]

11. Vanoli, E.; Schwartz, P.J. Sympathetic-parasympathetic interaction and sudden death. Basic Res. Cardiol. 1990, 85 (Suppl. 1), 305-321. [CrossRef]

12. Buch, A.N.; Coote, J.H.; Townend, J.N. Mortality, cardiac vagal control and physical training-What's the link? Exp. Physiol. 2002, 87, 423-435. [CrossRef] [PubMed]

13. Corrado, D.; Migliore, F.; Basso, C.; Thiene, G. Exercise and the risk of sudden cardiac death. Herz 2006, 31, 553-558. [CrossRef]

14. Oshima, Y.; Shiga, T.; Moritani, T.; Masuda, I.; Hayashi, T.; Nakao, K. Determination of optimal exercise intensity based on real-time analysis of heart rate variability during exercise. Jpn. J. Phys. Fit. Sports Med. 2003, 52, 295-304. [CrossRef]

15. Millet, G.P.; Roels, B.; Schmitt, L.; Woorons, X.; Richalet, J.-P. Combining hypoxic methods for peak performance. Sports Med. 2010, 40, 1-25. [CrossRef] [PubMed]

16. Favret, F.; Richalet, J.-P. Exercise and hypoxia: The role of the autonomic nervous system. Respir. Physiol. Neurobiol. 2007, 158, 280-286. [CrossRef] 
17. West, J.B.; Schoene, R.B.; Luks, A.M.; Milledge, J.S. High Altitude Medicine and Physiology, 5th ed.; Taylor \& Francis: Boca Raton, FL, USA, 2013. [CrossRef]

18. Saito, S.; Tanobe, K.; Yamada, M.; Nishihara, F. Relationship between arterial oxygen saturation and heart rate variability at high altitudes. Am. J. Emerg. Med. 2005, 23, 8-12. [CrossRef]

19. Oliveira, A.L.M.B.; de Azeredo Rohan, P.; Gonçalves, T.R.; da Silva Soares, P.P. Effects of hypoxia on heart rate variability in healthy individuals: A systematic review. Int. J. Cardiovasc. Sci. 2017, 30, 251-261. [CrossRef]

20. Lizamore, C.A.; Hamlin, M.J. The use of simulated altitude techniques for beneficial cardiovascular health outcomes in nonathletic, sedentary, and clinical populations: A literature review. High Alt. Med. Biol. 2017, 18, 305-321. [CrossRef]

21. Burtscher, M.; Ponchia, A. The risk of cardiovascular events during leisure time activities at altitude. Prog. Cardiovasc. Dis. 2010, 52, 507-511. [CrossRef]

22. Rimoldi, S.F.; Sartori, C.; Seiler, C.; Delacrétaz, E.; Mattle, H.P.; Scherrer, U.; Allemann, Y. High-altitude exposure in patients with cardiovascular disease: Risk assessment and practical recommendations. Prog. Cardiovasc. Dis. 2010, 52, 512-524. [CrossRef]

23. Bobyleva, O.V.; Glazachev, O.S. Changes in autonomic response and resistance to acute graded hypoxia during intermittent hypoxic training. Hum. Physiol. 2007, 33, 199-206. [CrossRef]

24. Bille, K.; Figueiras, D.; Schamasch, P.; Kappenberger, L.; Brenner, J.I.; Meijboom, F.J.; Meijboom, E.J. Sudden cardiac death in athletes: The Lausanne Recommendations. Eur. J. Cardiovasc. Prev. Rehabil. 2006, 13, 859-875. [CrossRef] [PubMed]

25. Karvonen, M.J.; Kentala, E.; Mustala, O. The effects of training on heart rate: A longitudinal study. Ann. Med. Exp. Biol. Fenn. 1957, 35, 307-315. [PubMed]

26. McNulty, C.R.; Robergs, R.A. New methods for processing and quantifying $\mathrm{VO}_{2}$ kinetics to steady state: $\mathrm{VO}_{2}$ onset kinetics. Front. Physiol. 2017, 8, 740. [CrossRef]

27. Caminal, P.; Sola, F.; Gomis, P.; Guasch, E.; Perera, A.; Soriano, N.; Mont, L. Validity of the Polar V800 monitor for measuring heart rate variability in mountain running route conditions. Eur. J. Appl. Physiol. 2018, 118, 669-677. [CrossRef] [PubMed]

28. Lippman, N.; Stein, K.M.; Lerman, B.B. Comparison of methods for removal of ectopy in measurement of heart rate variability. Am. J. Physiol. 1994, 267 Pt 2, H411-H418. [CrossRef]

29. Borg, G. Borg's Perceived Exertion and Pan Scales; Human Kinetics: Champaign, IL, USA, 1998.

30. Botek, M.; Stejskal, P.; Krejci, J.; Jakubec, A.; Gaba, A. Vagal threshold determination: Effect of age and gender. Int. J. Sports Med. 2010, 31, 768-772. [CrossRef]

31. Hautala, A.J.; Mäkikallio, T.H.; Seppänen, T.; Huikuri, H.V.; Tulppo, M.P. Short-term correlation properties of R-R interval dynamics at different exercise intensity levels. Clin. Physiol. Funct. Imaging 2003, 23, 215-223. [CrossRef]

32. Iwasaki, K.; Ogawa, Y.; Aoki, K.; Saitoh, T.; Otsubo, A.; Shibata, S. Cardiovascular regulation response to hypoxia during stepwise decreases from $21 \%$ to $15 \%$ inhaled oxygen. Aviat. Space Environ. Med. 2006, 77, 1015-1019.

33. Siebenmann, C.; Ryrsø, C.K.; Oberholzer, L.; Fisher, J.P.; Hilsted, L.M.; Rasmussen, P.; Secher, N.H.; Lundby, C. Hypoxia-induced vagal withdrawal is independent of the hypoxic ventilatory response in men. J. Appl. Physiol. 2019, 126, 124-131. [CrossRef]

34. Marshall, J.M. Chemoreceptors and cardiovascular control in acute and chronic systemic hypoxia. Braz. J. Med. Biol. Res. 1998, 31, 863-888. [CrossRef] [PubMed]

35. Rojas-Camayo, J.; Mejia, C.R.; Callacondo, D.; A Dawson, J.; Posso, M.C.; Galvan, C.A.; Davila-Arango, N.; Bravo, E.A.; Loescher, V.Y.; Padilla-Deza, M.M.; et al. Reference values for oxygen saturation from sea level to the highest human habitation in the Andes in acclimatised persons. Thorax 2018, 73, 776-778. [CrossRef] [PubMed]

36. Woorons, X.; Mollard, P.; Pichon, A.; Lamberto, C.; Duvallet, A.; Richalet, J.-P. Moderate exercise in hypoxia induces a greater arterial desaturation in trained than untrained men. Scand. J. Med. Sci. Sports 2007, 17, 431-436. [CrossRef] [PubMed] 
37. Wilber, R.L. Altitude Training and Athletic Performance; Human Kinetics: Champaign, IL, USA, 2004.

38. Van De Borne, P.; Montano, N.; Narkiewicz, K.; Degaute, J.P.; Malliani, A.; Pagani, M.; Somers, V.K. Importance of ventilation in modulating interaction between sympathetic drive and cardiovascular variability. Am. J. Physiol. Heart Circ. Physiol. 2004, 280, H722-H729. [CrossRef]

39. Shiraishi, Y.; Katsumata, Y.; Sadahiro, T.; Azuma, K.; Akita, K.; Isobe, S.; Yashima, F.; Miyamoto, K.; Nishiyama, T.; Tamura, Y.; et al. Real-time analysis of the heart rate variability during incremental exercise for the detection of the ventilatory threshold. J. Am. Heart Assoc. 2018, 7, e006612. [CrossRef]

Publisher's Note: MDPI stays neutral with regard to jurisdictional claims in published maps and institutional affiliations.

(C) 2020 by the authors. Licensee MDPI, Basel, Switzerland. This article is an open access article distributed under the terms and conditions of the Creative Commons Attribution (CC BY) license (http://creativecommons.org/licenses/by/4.0/). 